\title{
Benign Metastasising Leiomyoma: Is it a Real Threat nfiter Morcillation?
}

\author{
Kale Shriniketan S, More Sagar J \\ Department of Surgical Oncology, Navjeevan Hospital, Sangli and Department \\ of Pathology, Deesha Pathology Laboratory, Sangli.
}

\section{Abstract:}

Benign metastasising leiomyoma is a rare entity in which multiple benign fibroid tumors develop along the peritoneum. Due to its temporal relationship with laparoscopic hysterectomy particularly when morcillator was used to extract the specimen, it becomes interesting to understand whether this condition occurs due to implantation of the benign tumor cells. Here we present a case where post-laparoscopic hysterectomy, multiple benign fibroids have developed along the peritoneal cavity.

Key words: Leiomyoma, Hysterectomy, Neoplasms, Peritoneum, Endoscopy, Humans.

\section{Introduction}

Laparoscopic hysterectomy for fibroid uterus using morcillator is now being done quite commonly. This is a case where we have encountered multiple fibroids developing in the peritoneum 2 years after the laparoscopic hysterectomy. This is a rare situation and only handful of cases are been reported in the literature.

\section{Case Report}

45 year lady presented with a lump palpable over left lower abdomen noticed since 2 months. There was no pain associated with the swelling. She had a history of laparoscopic hysterectomy done two years back. On examination there was a firm swelling palpable in left iliac fossa region. There was no other palpable lump. All her haematological and biochemical investigations were normal. Her
CA 125 was 23 (within normal range). Her contrast enhanced computerised tomography scan (CT scan) showed multiple, isodense, homogeneously enhancing masses in mesentery in umbilical and infra-umbilical region [Fig.1]. Largest mass was seen in pelvis of size $88 \times 57 \times 85 \mathrm{~mm}$. There was a mass noted in left paraumbilical region of size $57 \times 32 \mathrm{~mm}$. Both the ovaries were noted normal with a cyst of size $29 \times 26 \mathrm{~mm}$ in right ovary. Rest of the abdominal organs were normal. Fine needle aspiration cytology of the abdominal mass showed a tumor of smooth muscle origin. So a diagnosis of GIST or leiomyosarcoma was kept and patient was explored.

On exploration, multiple masses were located in pouch of Douglus, in retroperitoneum and in the anterior abdominal wall. There was a similar

\section{Corresponding Author: Dr. Kale Shriniketan S.}

Email: shrinkale@yahoo.co.in

Received: December 16, 2014 | Accepted: February 10, 2015 | Published Online: February 28, 2015 This is an Open Access article distributed under the terms of the Creative Commons Attribution License (creativecommons.org/licenses/by/3.0) Conflict of interest: None declared | Source of funding: Nil | DOl: http://dx.doi.org/10.17659/01.2015.0023 
nodular mass in the omentum. All lesions were excised along with right ovary. Grossly all excised masses had similar appearance [Fig.2]. It had grey tan firm appearance on cut section. Similarly right ovary also was firm grey with tiny cystic spaces within the masses.

Microscopically all the masses showed elongated spindle shaped cells with blunt ended elongated nuclei. There cells were arranged in fascicles. Interspersed amongst them were mononuclear cells. There was myxoid degeneration at places. There was no evidence of malignancy in the specimen. Immunohistochemistry was done which showed the tumor to be C-kit negative.

Excised ovary showed cystic follicles and multiple glands lined by tall columnar epithelium. Glands are filled with mucin and macrophages. Mononuclear cell were seen infiltrating the glands. Hence, the diagnosis of benign metastasising leoimyoma (BML) with mucinous cystadenofibroma of the ovary was confirmed.

On retrieving the record of total laparoscopic hysterectomy done 2 years back, it was evident that myomectomy was done and the morcillator was used to fragment the specimen. So the tumour implantation as possible aetiology for the disease was entertained.

\section{Discussion}

Uterine leiomyoma is one of the commonest benign tumors we encounter in our practice. One of the rare sites for the occurrence of leiomyoma is peritoneum. Essentially there is no smooth muscle in peritoneum however the development of leiomyoma can still occur in the peritoneum. This is due to smooth muscle metaplasia of the subperitoneal mesenchyme. This condition is labelled as disseminated leiomyomatosis peritonei [1].

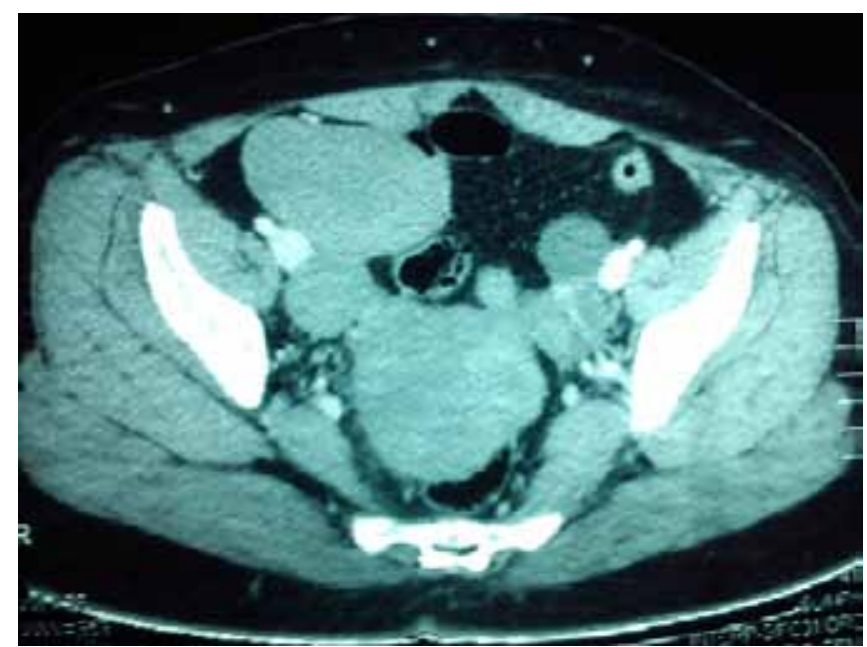

Fig.1: CT scan showing multiple, isodense, homogeneously enhancing masses in mesentery.

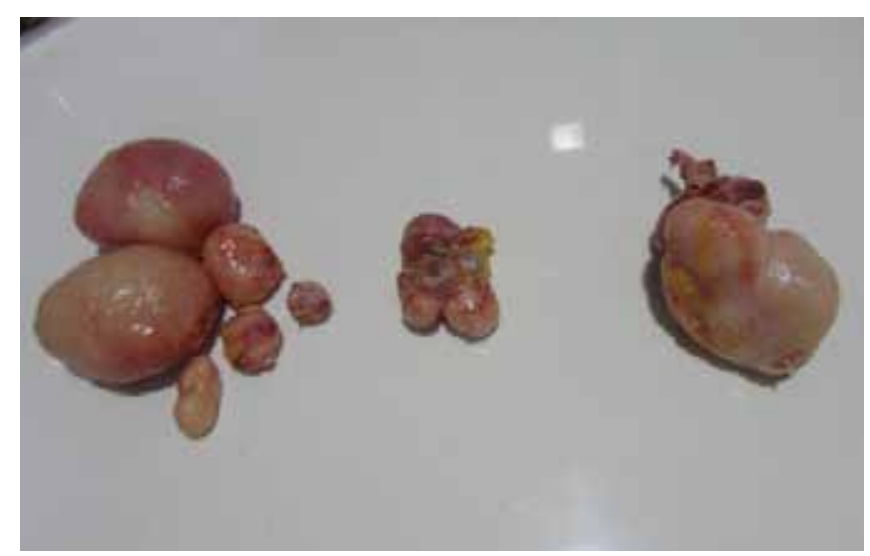

Fig.2: Multiple tumor specimen seen on excision.

The second and more common explanation for this condition could be tumor implantation that occurs during morcillation of the specimen. During the process there is a possibility that the cells from the tumor are showered into the peritoneum. These cells get implanted at various locations and grow there to form parasitic leiomyomas $[1,2]$. An article published by Takeda et al. has exploited this temporal relationship between the laparoscopic surgery and the development of multiple peritoneal leiomyomata [3]. In current case also laparoscopic 
hysterectomy was done for large uterine leiomyoma two years back when morcillation was done. So this occurrence of leiomyomas can be explained by tumor implantation theory.

Interesting thing about these tumors is that, though they are histologically non-malignant and have a tendency to metastasise systemically. Lung is the commonest site for metastasis. Such cases have been reported sporadically in the literature [4]. In our case there was no metastasis outside abdomen. It is very important to differentiate these tumors from leiomyosarcoma and smooth muscle tumors of uncertain malignant potential (STUMP) based on features like nucear atypia, necrosis and mitosis. As all the tumors in the said case don't have any of these features histologically hence they are classified as BML [4].

Benign metastasising leiomyoma is a real entity which may happen secondary to use of morcillator. More careful morcillation to avoid spillage and thorough peritoneal lavage can help in reducing this rare complication.

\section{Conclusion}

Multiple fibroids developing along the peritoneum after the laparoscopic hysterectomy is a rare complication. It is a rare but real threat that needs to be kept in mind while doing morcilation.

\section{References}

1. Sinha R, Sundaram M, Lakhotia S. Recurrent Leiomyomatosis. Journal of Minimally Invasive Gynecology. 2009;16(6):667-668.

2. Michal B, Katarzyna RB, Beata W, Andrzej $D$, Tomasz R. Leiomyomatosis peritonealis disseminate with formation of endometrial cysts within tumors arising after supracervical laparoscopic hysterectomy. Ginekol Pol. 2013;84:68-71.

3. Takeda A, Mori M, Sakai K, Mitsui T, Nakmura H. Parasitic peritoneal leiomyomatosis diagnosed 6 years after laparoscopic myomectomy with electric tissue morcellation: Report of a case and review of the literature. Journal of Minimally Invasive Gynaecology. 2007;1 4:770-775.

4. Beck MM, Biswas B, D'Souza A, Kumar R. Pulmonary benign metastasizing leiomyoma from uterine leiomyoma. Hong Kong Med J. 2012; 18:153-155. 